\title{
Downregulation of CENPK suppresses lung adenocarcinoma by regulating EMT
}

Fengwen Xie, Bin Yuan, Jichun Liu

Department of Cardiovascular Surgery, The First Affiliated Hospital of Nanchang University, Nanchang, China

Submitted: 5 August 2020, Accepted: 28 May 2021

Online publication: 8 June 2021

Arch Med Sci

DOI: https://doi.org/10.5114/aoms/138261

Copyright $\odot 2021$ Termedia \& Banach

\section{Abstract}

Introduction: Centromere protein K (CENPK) plays a key role in regulating the assembly and function of centromeres, which in turn can affect the occurrence and development of various tumors. However, little is known about the biological function of CENPK in lung adenocarcinoma (LUAD).

Material and methods: We evaluated the relationship between CENPK expression and the clinicopathological characteristics of LUAD patients via a bioinformatics method based on data taken from the TCGA database. Then, the role of CENPK in LUAD was investigated in vitro by using the human LUAD cell line A549. Cell Counting Kit- 8 and colony formation assays were used detect the cell proliferation ability. Wound healing and transwell assays were used to detect the cell migration and invasion ability. Epithelial-mesenchymal transition (EMT) markers (E-cadherin, N-cadherin, Snail and vimentin) were measured by western blotting. In addition, a xenograft experiment was used to explore the function of CENPK in vivo.

Results: CENPK is highly expressed in LUAD tissues and cell lines. Moreover, high expression of CENPK in LUAD is significantly associated with stage, lymph node involvement and poor survival. CENPK knockdown significantly decreases the proliferation, migration and invasion ability of A549 cells by EMT both in vitro and in vivo.

Conclusions: CENPK is highly expressed in LUAD and leads to a poor prognosis. CENPK knockdown can suppress the proliferation, migration, and invasion of lung cancer cells via EMT, which may be a valid target for treatment.

Key words: bioinformatics, lung adenocarcinoma, CENPK.

\section{Introduction}

Lung cancer is one of the most common tumors worldwide, accounting for approximately $22 \%$ of cancer-related deaths, which makes it first among cancers in terms of mortality. In the US, lung cancer accounts for $13 \%$ of all tumor types, and approximately 135,000 people die from the disease each year [1]. An epidemiological survey conducted in 2015 in China recorded 730,000 lung cancer cases and 610,000 related deaths [2]. According to the World Lung Cancer Association, lung cancer can be divided into small cell lung cancer (SCLC) and non-small-cell lung cancer (NSCLC). NSCLC is the most common pathological type encountered in the clinic, accounting for $85 \%$ of new cases each year [3]. With the promotion of smoking bans, the pathological spectrum of NSCLC has changed dramatically; approximately $40-60 \%$ of new NSCLC cases are

\author{
Corresponding author: \\ Jichun Liu \\ Department \\ of Cardiovascular Surgery \\ The First Affiliated Hospital \\ of Nanchang University \\ Nanchang, China \\ E-mail: liujichun9999@126. \\ com
}


lung adenocarcinoma (LUAD), occurring especially among nonsmoking women in East Asia [4]. LUAD originates from small alveolar epithelial cells of the type II airways and is characterized by easy recurrence and metastasis [5]. The disease stages of LUAD play an important guiding role in formulating specific treatment plans for patients with LUAD. For the treatment of lung cancer, in addition to surgery, radiotherapy and chemotherapy, targeted therapy and immunotherapy have become the new development direction of lung cancer treatment. For example, studies on the third-generation epidermal growth factor receptor (EGFR) mutation inhibitor osimertinib show that the median progression-free survival (PFS) of lung cancer patients treated with osimertinib is 10.1 months, which is significantly longer than that of patients treated with chemotherapy (median PFS is 4.4 months) $[6,7]$. Immunotherapy was selected as the benchmark achievement of medical progress in 2016 by the American Society of Clinical Oncology (ASCO), which is the most cutting-edge in the field of cancer treatment [8]. At present, immunotherapeutic drugs for lung cancer are mainly immune checkpoint inhibitors, such as programmed cell death protein-1/programmed cell death ligand 1 (PD-1/PD-L1) [9]. A number of large-scale clinical trials have shown that compared with chemotherapy patients, the overall survival of lung cancer patients treated with PD-1/PD-L1 immune checkpoint inhibitors is significantly longer [10, 11]. Although technological progress has improved the diagnosis and treatment of LUAD, the 5 -year survival rate is only $10-15 \%$ [12]. Therefore, the identification of prognostic markers and therapeutic targets for LUAD is a topic of intense research in lung cancer research.

Centromere protein $\mathrm{K}$ (CENPK) belongs to the mitochondrial protein family and can be recruited to centromeres during cell division and participate in the processes of centromere assembly, mitosis and chromosome separation [13-15]. An increasing body of evidence has implicated dysregulation or dysfunction of CENPK in cancer progression. Research shows that CENPK is an oncogene that can promote cancer cell proliferation and metastasis in liver cancer and ovarian cancer [16-18]. Overexpression of CENPK was associated with poor outcomes in patients with triple-negative breast cancer [19]. However, the biological functions of CENPK in LUAD still remain unclear.

Epithelial-mesenchymal transition (EMT) refers to the transformation of differentiated epithelial cells into mesenchymal cells under specific physiological and pathological conditions, and is an inherent biological process of cells existing in various tissue types and development stages [20]. EMT is closely related to a variety of biological processes, including wound healing, fibrosis and cancer development [21]. According to the different physiological environments, EMT can be divided into three subtypes, including type 1 , type 2 and type 3 [22]. Type 3 is the most studied type at present, which is closely related to the growth and metastasis of malignant tumor cells [22]. A number of studies have shown that EMT occurs during the invasion and metastasis of lung cancer, liver cancer, breast cancer, and other tumors [23-25]. The main functions of EMT include [26]: (1) the loss of polarity of epithelial cells, the decrease of the ability of tight connection between cells, the long spindle shape and the loose arrangement of cells, similar to the characteristics of mesenchymal cells. (2) The changes of protein expression, down-regulation of the expression of E-cadherin, $\beta$-catenin, cytokeratin 8 (CK8), cytokeratin 18 (CK18), up-regulation of the expression of mesenchymal marker proteins, such as N-cadherin, vimentin and fibronectin. (3) When the tight connection between epithelial cells decreases and transforms into stromal cells, their biological behaviors change when their invasion ability is improved. Proteolytic enzymes degrade the cell basement membrane, allowing cells to invade the extracellular matrix. Therefore, the cells have a strong ability of invasion and metastasis. Once metastasis occurs, cancer cells can escape chemical treatment, which leads to the deteriorating prognosis of cancer patients [26].

In this study, the relationship between the expression of CENPK and LUAD was investigated using bioinformatics methods and in vitro/vivo experiments.

\section{Material and methods}

\section{The Cancer Genome Atlas (TCGA) database analysis}

The TCGA database (https://cancergenome.nih. gov/) is a research project jointly developed by the National Cancer Institute and the National Human Genome Research Institute that aims to discover gene mutations and signaling pathways related to tumor occurrence, development, and prognosis at the molecular level through genome-wide analysis of tumor specimens [27]. Transcript and clinical data from LUAD and normal tissue samples were obtained from the TCGA database, which is public and can be downloaded free of charge, with confirmation that all relevant informed consent has been supplied. Clinical information, including patient age and sex, disease stage, histological type, tumor-node-metastasis (TNM) classification, follow-up time, and survival status, was extracted from the data of 515 LUAD patients, from which 515 tumor tissues and 59 adjacent normal tissues were obtained. 


\section{Oncomine database analysis}

The Oncomine database (https://www.oncomine.org/) is currently the world's largest tumor gene chip database and integrated data mining platform and can be used to mine cancer gene-related information [28]. The mRNA expression level of CENPK in LUAD and other cancers was detected through Oncomine database analysis. $P$-value < 0.05 , fold change $>2$ and top $10 \%$ gene rank were selected as thresholds for comparisons between tumor and normal samples.

\section{PrognoScan database analysis}

PrognoScan (http://www.prognoscan.org/) is a data-base used to explore the relationship between gene expression and the prognosis of patients across a large collection of publicly available cancer microarray datasets [29], and this database was applied to analyze the prognostic value of CENPK in LUAD patients. The threshold was adjusted to a Cox $p<0.05$.

\section{Cell culture}

The human LUAD cell lines A549, PC9, H1299, and $\mathrm{H} 1975$ and the normal human lung epithelial cell line BEAS-2B were purchased from the Shanghai Institute of Cell Biology, Chinese Academy of Sciences. They were cultured in Roswell Park Memorial Institute (RPMI, Thermo Scientific, USA) 1640 medium with $10 \%$ fetal bovine serum (FBS, Gibco, USA) and 1\% antibiotics (penicillin-streptomycin, Sangon, China) at $37^{\circ} \mathrm{C}$ in a $5 \% \mathrm{CO}_{2}$ incubator. Both of the cell lines were sub-passaged for fewer than 6 months.

\section{Cell transfection}

Small interfering RNA (siRNA) targeting CENPK and a negative control (NC) siRNA were purchased from GenePharma (Shanghai). The oligonucleotides were transfected using Lipofectamine 2000 (Invitrogen, USA) according to the manufacturer's instructions. The A549 cells were seeded into sixwell plates one day before transfection. After cell confluence reached $90-95 \%, 10 \mu$ l of DNA was diluted with $100 \mu \mathrm{l}$ of serum-free medium, and then another $100 \mu \mathrm{l}$ of serum-free medium was used to dilute $5 \mu \mathrm{l}$ of Lipofectamine 2000 transfection reagent. Subsequently, the solution was mixed with the diluted DNA at room temperature for $20 \mathrm{~min}$ and added to each well. The sequences of SiRNA-1, SiRNA-2, siRNA-3 and NC were $5^{\prime}$-UCCCUUGACUGAAGACGUUTT-3', 5'-GCUCAGCUAUCAUUGUUAATT-3', 5'-CUGCCUGAUAGAAGUGUUATT-3', and 5'-UUCUCCGAACGUGUCACGUTT-3', respectively. The transfection efficiency was detected by Western blotting.

\section{Quantitative real-time PCR (qRT-PCR)}

Total RNA was extracted from cells using TRIzol reagent (Invitrogen) and reverse-transcribed to cDNA using the EasyScript First-Strand cDNA Synthesis SuperMix Kit (TransGen Biotech). QRT-PCR was performed with the StepOnePlus Real-Time PCR System (Applied Biosystems) and Fast Start Universal SYBR Green Master Mix (Takara). The relative quantification of CENPK to the internal control (GAPDH) was calculated using the 2- $-\Delta \Delta c t$ method. The primers for qPCR are listed in Table I.

\section{Western blotting}

Western blotting was performed according to a previous description [30]. Briefly, whole cell lysates were normalized by OD600. Total protein was extracted using RIPA lysis buffer (Beyotime, Shanghai, China). The protein concentration was detected using a BCA assay (Thermo Scientific, USA). After SDS-PAGE and blotting, the membrane was incubated with primary antibodies overnight at $4^{\circ} \mathrm{C}$. Then, the membrane was incubated with the corresponding secondary antibodies for $2 \mathrm{~h}$. The following primary antibodies were used: CENPK (1 : 500, ProteinTech, Wuhan, China), GAPDH (1 : 1000, Goodhere, Hangzhou, China), E-cadherin (1 : 2000, ProteinTech, Wuhan, China), N-cadherin (1 : 2000, ProteinTech, Wuhan, China), Snail (1 : 500, ProteinTech, Wuhan, China), and vimentin (1 : 1000, ProteinTech, Wuhan, China). GAPDH was utilized as an internal control. The corresponding secondary antibodies were used: anti-rabbit IgG-horseradish peroxidase or anti-mouse IgG-HRP (HRP, 1 : 5,000; Boster, Wuhan, China). The signals were detected by enhanced chemiluminescence (Thermo Scientific, USA). The gray values of bands were measured by QuantityOne software (Bio-Rad), and the relative protein abundance was normalized to GAPDH expression.

\section{Cell Counting Kit-8 (CCK-8) assay}

Forty-eight hours after transfection, A549 cells were added to a 96-well plate (approximately 2000 per well) and incubated for 0, 24, 48, and $72 \mathrm{~h}$; then, $10 \mu \mathrm{l}$ of CCK-8 reagent (Beyotime Biotechnology, Shanghai, China) was added to each well. The plate was incubated in a $37^{\circ} \mathrm{C}$ incubator for $4 \mathrm{~h}$, and the absorbance value was detected at a wavelength of $450 \mathrm{~nm}$ with a microplate reader.

Table I. Primer sequences used in the qRT-PCR

\begin{tabular}{|llc|}
\hline Name & Type & Sequence $\left(\mathbf{5}^{\prime} \mathbf{-} \mathbf{3}^{\prime}\right.$ ) \\
\hline CENPK & Forward & CAGATGTGGGAGATGTTAC \\
\cline { 2 - 3 } & Reverse & CATTTGAATCGGTGAGTGT \\
\hline \multirow{2}{*}{ GAPDH } & Forward & TCAAGAAGGTGGTGAAGCAGG \\
\cline { 2 - 3 } & Reverse & TCAAAGGTGGAGGAGTGGGT \\
\hline
\end{tabular}




\section{Colony formation assay}

The transfected A549 cells were seeded in sixwell plates at 300 cells per well for colony formation assays following the methods described previously [30]. Briefly, the A549 cells were seeded into 6-well plates (approximately 200 per well) $48 \mathrm{~h}$ after transfection. After culture for another 2 weeks, the colonies were fixed with paraformaldehyde (4\%, $30 \mathrm{~min}$ ) and stained with crystal violet $(0.5 \%, 30 \mathrm{~min})$ at room temperature. The number of colonies was counted under the light microscope.

\section{Wound healing assay}

The transfected A549 cells were plated into a 6-well plate. When the cells were approximately $70 \%$ confluent, the petri dish was scratched with a $10-\mu l$ pipette tip, rinsed with PBS 3 times, serum-free medium was added, and the cells were cultured for $24 \mathrm{~h}$. The width of the scratch gap was observed and measured using an inverted microscope.

\section{Transwell assay}

For cell invasion detection, a Transwell assay was adopted according to a previous description [30]. In brief, the treated A549 cells were plated on the upper part of the Transwell chamber (precoated with Matrigel) in serum-free RPMI-1640, and medium with $10 \%$ FBS was added to the lower part of the chamber as a chemoattractant. After incubation for $24 \mathrm{~h}$ at $37^{\circ} \mathrm{C}$, cells in the upper part of the chamber were removed. Migrated cells in the lower part of the chamber were analyzed by $0.2 \%$ crystal violet staining with an inverted microscope. The cell population was counted and calculated in five random visual fields of each chamber.

\section{Construction of lentiviral vectors of CENPK shRNA}

To obtain CENPK shRNA plasmid, the targeting sequences specific for CENPK (5'-GCGAGTTTCTAGAAGACC-3') were constructed into a lentiviral vector and a scrambled shRNA lentiviral vector was constructed as a negative control. The CENPK coding sequence was amplified using PCR and ligated into a lentiviral vector. Prepared lentiviral particles were transfected into A549 cells, and puromycin was used to select positive cells.

\section{Xenograft tumor in nude mice}

The animal protocol was approved by the Institutional Animal Care and Use Committee of the First Affiliated Hospital of Nanchang University (Jiangxi, China). Ten 5-week-old female nude mice were purchased from the animal house of the
Faculty of Medicine, Nanchang University (Jiangxi, China), and were randomly divided into two equal groups. $200 \mu \mathrm{l}$ of A549 cell suspension $\left(2 \times 10^{6} / \mathrm{ml}\right)$ which was transfected with shNC or shCENPK was subcutaneously injected into the right flank of mice in both groups. Tumor volumes were examined every 3 days using the formula: volume $=0.5$ $\times$ length $\times$ width $^{2}$. At 30 days after the subcutaneous injection, mice were sacrificed and tumors were isolated and weighed.

\section{Statistical analysis}

Statistical analysis was performed using GraphPad Prism 8.0 (Intuitive Software for Science, San Diego, CA, USA). Data are expressed as the mean \pm standard deviation. Student's $t$-test was employed for comparisons between two groups, while oneway analysis of variance (ANOVA) was performed for multiple group comparisons. Each experiment was repeated three times independently. A $p<$ 0.05 was considered statistically significant.

\section{Results}

\section{CENPK is upregulated in LUAD samples and associated with poor prognosis}

The study samples comprised 59 healthy patients and 515 tumor samples from the TCGA database. CENPK was significantly upregulated in LUAD samples compared with healthy patients ( $p<$ 0.001; Figure 1). In addition, we further confirmed the high expression of CENPK in LUAD samples using the Oncomine database (Figure 2). Increased CENPK expression was significantly associated with disease stage $(p<0.017)$ and $N(p=0.024)$ classification (Figure 3). The Kaplan-Meier curves revealed a worse prognosis for the high CENPK expression group than for the low expression group $(p<0.001$; Figure 4). In addition, the PrognoScan database revealed that high expression of CENPK was negatively correlated with overall survival and relapse-free survival in LUAD patients (Figure 5). Moreover, expression of CENPK was significantly associated with worse OS in the univariate Cox regression analysis (hazard ratio $(H R)=1.54 ; 95 \%$ confidence interval (Cl): 1.19-1.99; $p<0.001$; Table II). Multivariate Cox regression analysis indicated that CENPK is an independent predictor of prognosis for LUAD patients $(\mathrm{HR}=1.60 ; 95 \% \mathrm{Cl}$ : 1.22-2.11; $p<0.001$; Table II).

\section{High expression of CENPK is inhibited by SiRNA}

QRT-PCR and western blotting results showed that the $\mathrm{mRNA}$ and protein expression levels of CENPK were all significantly higher in human LUAD cell lines (A549, PC9, H1299, H1975) than 
A

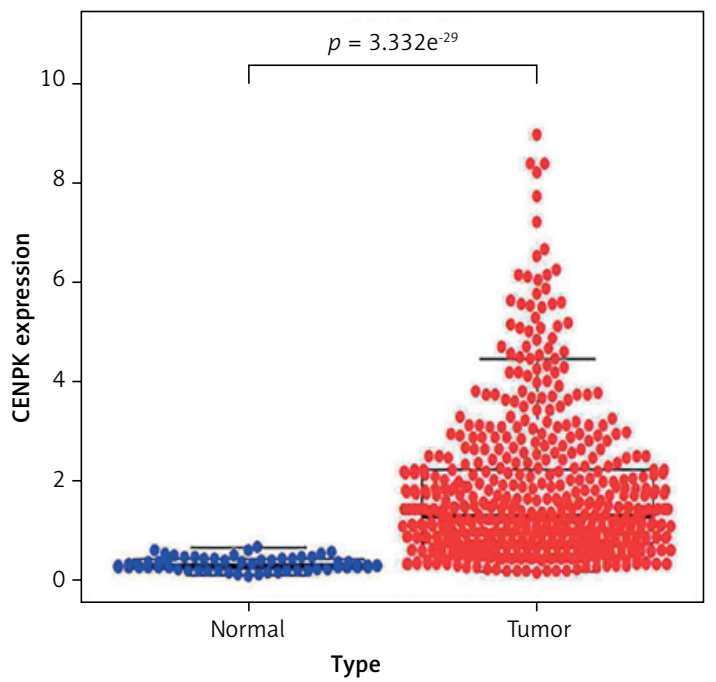

B

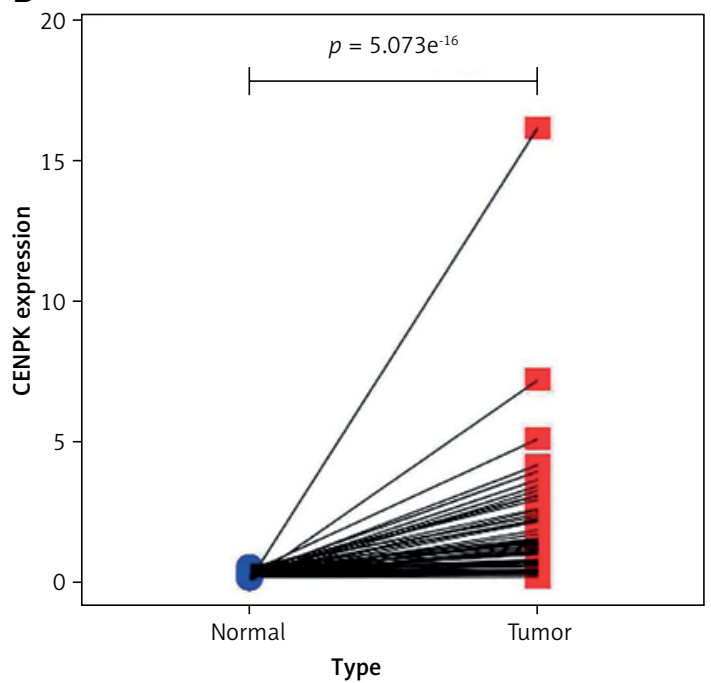

Figure 1. CENPK expression was significantly higher in LUAD samples compared with healthy patients from the TCGA database (A - unpaired $t$-test, $p<0.001 ; \mathbf{B}$ - paired $t$-test, $p<0.001$ )

A

\begin{tabular}{|c|}
\hline Analysis type by cancer \\
\hline Bladder cancer \\
\hline Brain and CNS cancer \\
\hline Breast cancer \\
\hline Cervical cancer \\
\hline Colorectal cancer \\
\hline Esophageal cancer \\
\hline Gastric cancer \\
\hline Head and neck cancer \\
\hline Kidney cancer \\
\hline Leukemia \\
\hline Liver cancer \\
\hline Lung cancer \\
\hline Lymphoma \\
\hline Melanoma \\
\hline Myeloma \\
\hline Other cancer \\
\hline Ovarian cancer \\
\hline Pancreatic cancer \\
\hline Prostate cancer \\
\hline Sarcoma \\
\hline Significant unique analyses \\
\hline Total unique analyses \\
\hline
\end{tabular}

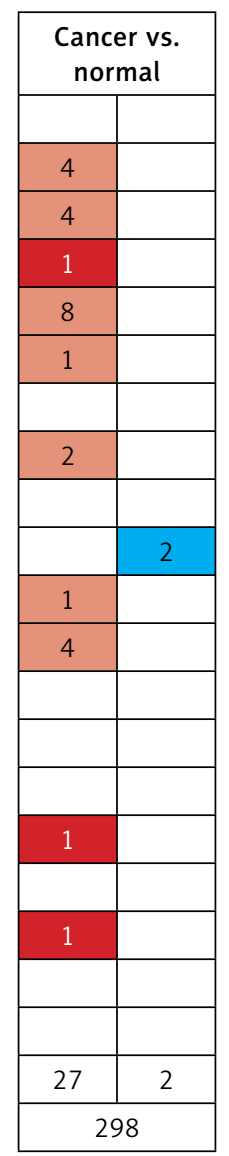

B
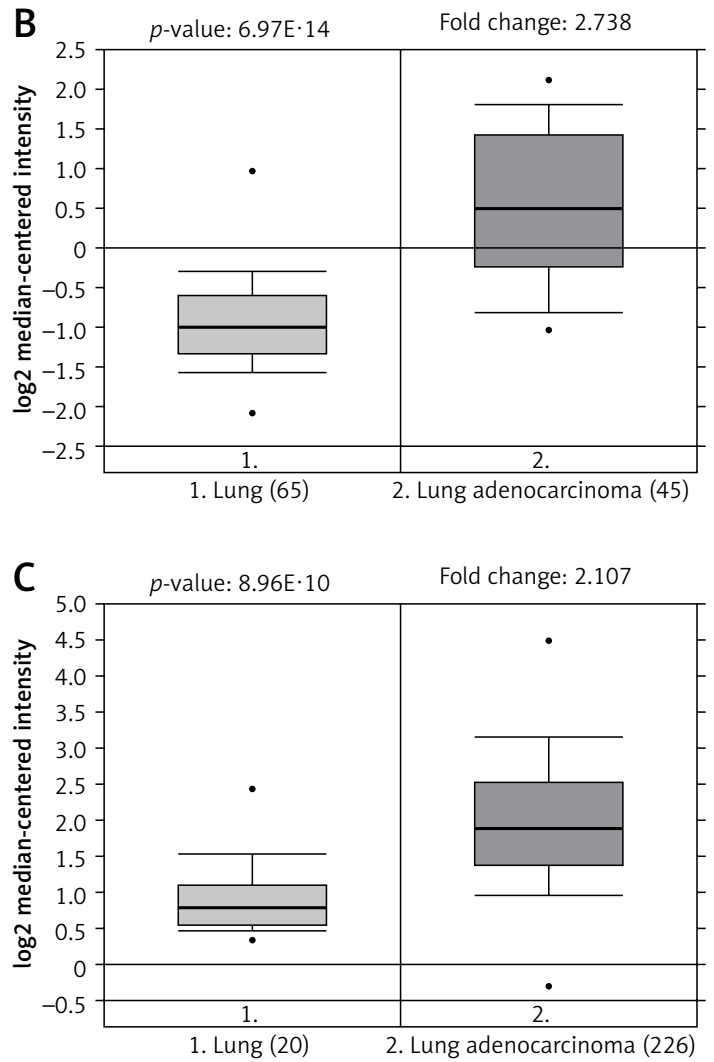
$\stackrel{15101051}{\longrightarrow}$

Figure 2. Expression level of CENPK in LUAD (Oncomine database). A - Expression level of CENPK in different tumors. The red left column shows the number of samples with CENPK upregulation, and the blue right column shows the number of samples with CENPK downregulation. B-D - Box plot comparing CENPK expression in normal and LUAD tissues. $\mathbf{E}$ - Meta-analysis of CENPK expression in 3 analyses 


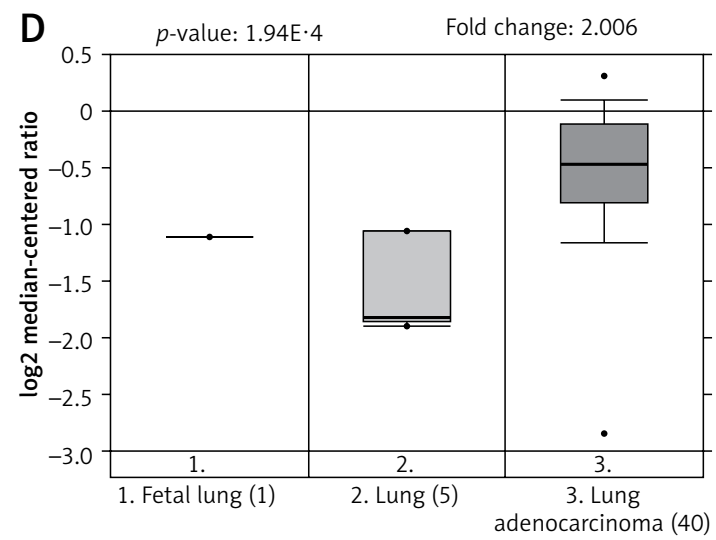

E Comparison of CENPK Across 3 analyses Over-expression

\begin{tabular}{|c|c|c|c|c|c|}
\hline Median rank & $p$-value & Gene & & & \\
\hline 384.0 & $1.94 \mathrm{E}-4$ & CENPK & & & \\
\hline & & & 1 & 2 & 3 \\
\hline
\end{tabular}

Legend

1. Lung adenocarcinoma vs. normal Garber Lung, Proc Nati Acad Sci USA, 2001

2. Lung adenocarcinoma vs. normal

Hou Lung, PLoS One, 2010

3. Lung adenocarcinoma vs. normal

Okayama Lung, Cancer Res, 2012

$151025 \quad 251051$

\section{$\stackrel{\square \square \square \square \square \square \square \square}{\stackrel{2}{\longleftarrow}}$}

The rank for a gene is the median rank for that gene across each of the analyses.

The $p$-value for a gene is its $p$-value for the median-ranked analysis.

Figure 2. Cont. D - Box plot comparing CENPK expression in normal and LUAD tissues. E - Meta-analysis of CENPK expression in 3 analyses

A

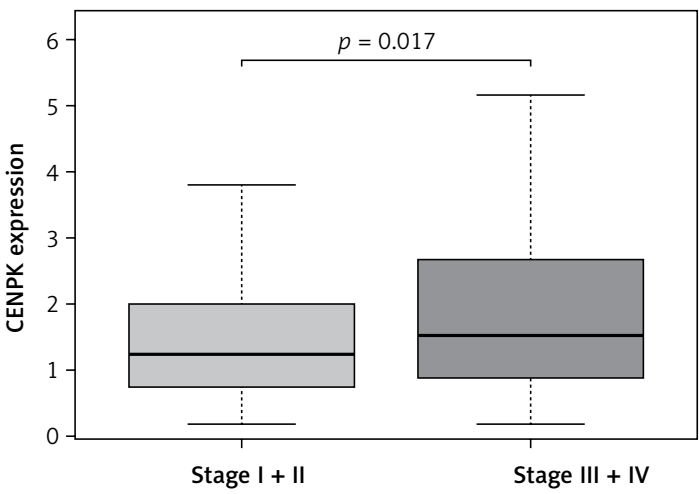

B

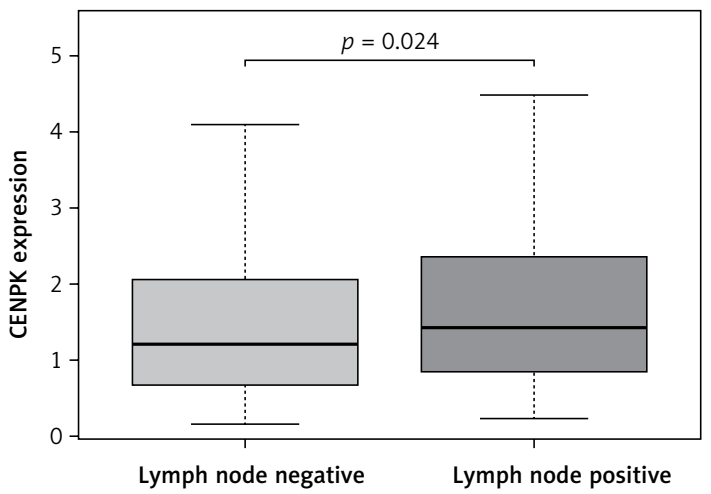

Figure 3. Associations between expression level of CENPK and clinicopathological characteristics of LUAD patients (TCGA database). A - Patients with a more aggressive disease stage have significantly higher CENPK expression. B - Patients with lymph node metastasis have higher CENPK expression. $P<0.01$

A

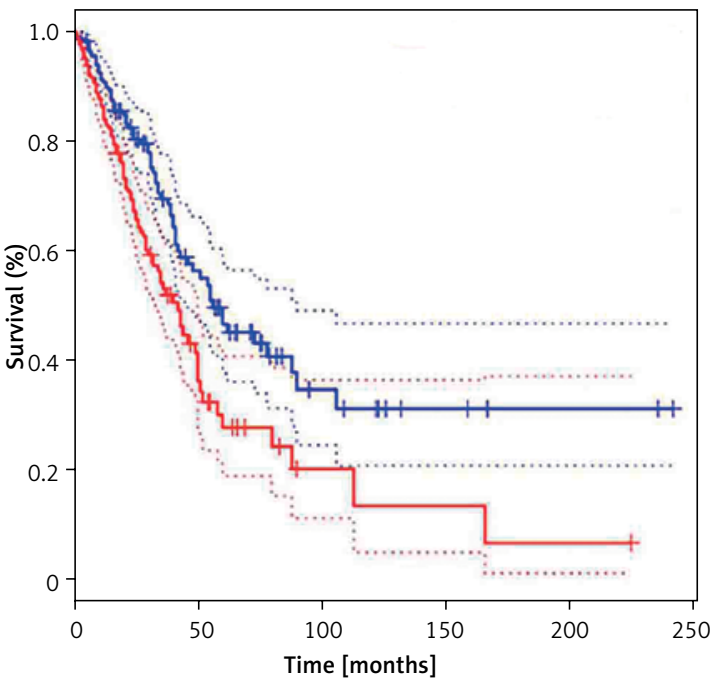

- Low CENPK group $\quad$ - High CENPK group Logrank $p=0.00039$
B

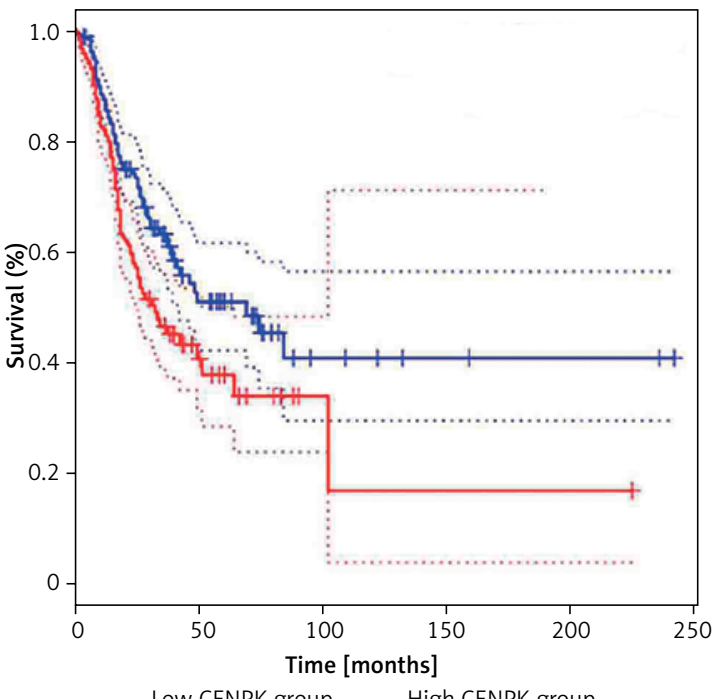

- Low CENPK group $\quad-$ High CENPK group Logrank $p=0.0037$

Figure 4. Kaplan-Meier curves for survival probability in LUAD patients (TCGA database). A - High CENPK expression was related to poor overall survival. B - High CENPK expression was related to poor disease-free survival. $P<0.01$ 
A Lung adenocarcinoma, GSE13213, overall survival

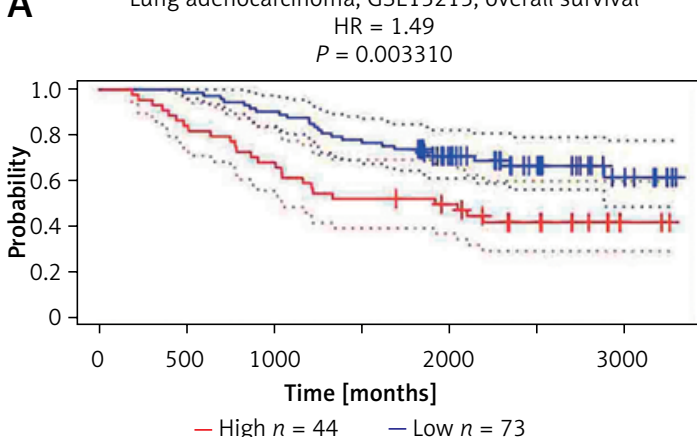

B Lung adenocarcinoma, GSE31210, relapse-free survival

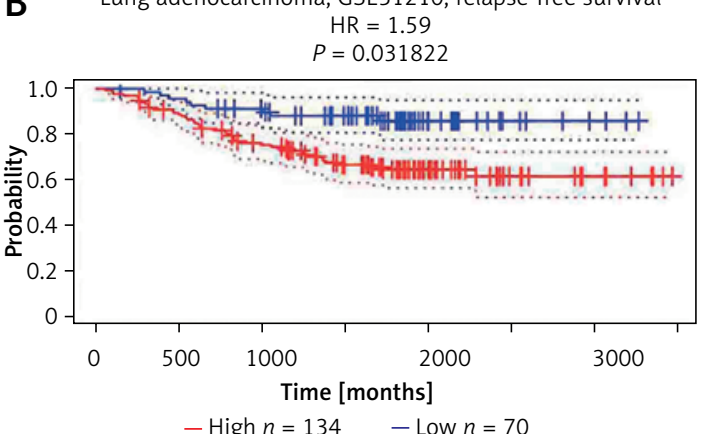

- High $n=134 \quad-$ Low $n=70$

Figure 5. Kaplan-Meier curves for survival probability in LUAD patients (PrognoScan database). A - High CENPK expression was related to poor overall survival. B - High CENPK expression was related to poor relapse-free survival. $P<0.05$

Table II. Univariate analysis and multivariate analysis of the correlation of CENPK expression with OS among lung adenocarcinoma patients from the TCGA database. Early stage: stages I and II; late stage: stages III and IV

\begin{tabular}{|lcccccc|}
\hline Parameter & \multicolumn{3}{c}{ Univariate analysis } & \multicolumn{3}{c|}{ Multivariate analysis } \\
\cline { 2 - 7 } & HR & $\mathbf{9 5 \% ~ C l}$ & $P$-value & HR & $95 \% \mathrm{Cl}$ & $P$-value \\
\hline Age (<60 vs. $\geq 60)$ & 1.03 & $0.68-1.56$ & 0.88 & 1.16 & $0.76-1.77$ & 0.48 \\
\hline Sex (female vs. male) & 1.04 & $0.72-1.49$ & 0.85 & 0.91 & $0.63-1.32$ & 0.62 \\
\hline $\begin{array}{l}\text { Pathological stage } \\
\text { (early stage vs. late stage) }\end{array}$ & 2.94 & $2.02-4.29$ & $<0.001$ & 1.46 & $0.84-2.52$ & 0.18 \\
\hline T (T1+ T2 vs. T3+ T4) & 2.66 & $1.69-4.18$ & $<0.001$ & 1.90 & $1.13-3.20$ & 0.02 \\
\hline M (MO vs. M1) & 1.76 & $0.96-3.20$ & 0.07 & 1.15 & $0.58-2.30$ & 0.69 \\
\hline N (N0 vs. N1 + N2+ N3) & 2.86 & $1.97-4.14$ & $<0.001$ & 2.23 & $1.42-3.50$ & $<0.001$ \\
\hline CENPK expression (low vs. high) & 1.54 & $1.19-1.99$ & $<0.001$ & 1.60 & $1.22-2.11$ & $<0.001$ \\
\hline
\end{tabular}

in the normal human lung epithelial cell line (BEAS-2B) (Figure 6). Among them, the expression of CENPK in the A549 cell line was higher than that in other human LUAD cell lines. Therefore, the

A

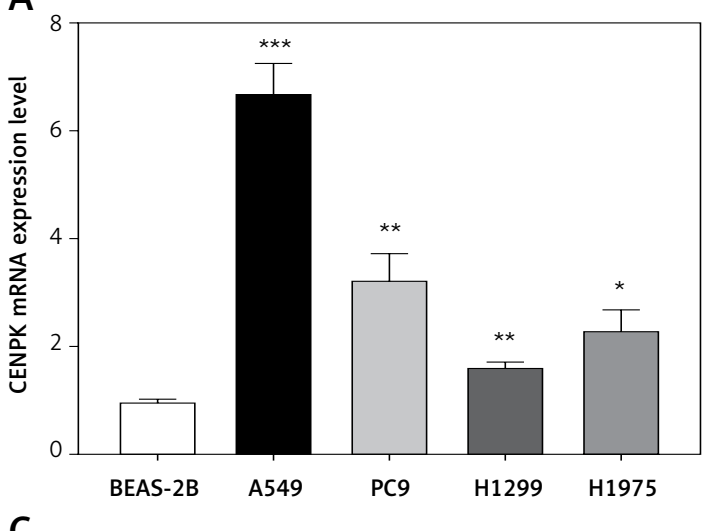

C

BEAS-2B

A549

PC9

H1299

H1975

CENPK

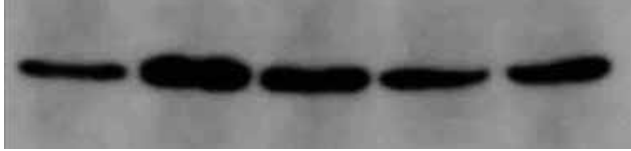

GAPDH

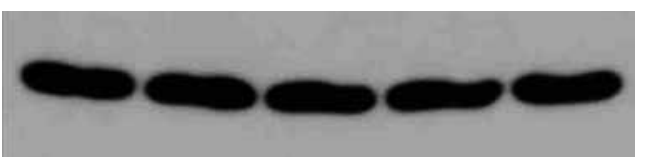

A549 cell line was used for the following experiment. siRNAs (siRNA-1, siRNA-2, siRNA-3) were transfected into the $A 549$ cell line, and the protein expression levels of CENPK were all decreased,

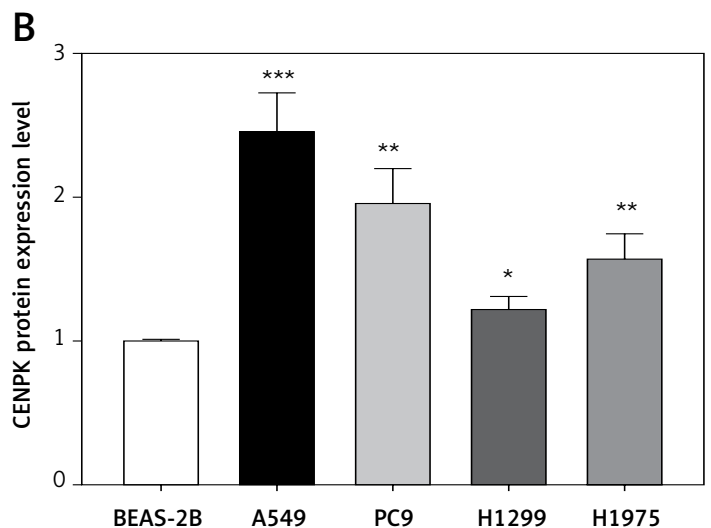

Figure 6. CENPK is upregulated in LUAD cells. A - CENPK is upregulated in human LUAD cell lines (A549, PC9, H1299, H1975) at the mRNA level detected by qRT-PCR. B, C - CENPK is upregulated in human LUAD cell lines (A549, PC9, H1299, H1975) at the protein level detected by western blotting. ${ }^{\star} P<0.05 ;{ }^{* *} p<0.01 ;{ }^{* * *} p<0.001$ 
as detected by western blotting; siRNA-2 showed greater silencing efficiency than the other siRNAs (Figure 7). Hence, siRNA-2 was utilized for specific RNA interference in the following experiment.

\section{CENPK silencing inhibits proliferation of LUAD cells}

The effect of CENPK silencing on the proliferation ability of LUAD cells was examined by CCK-8

A

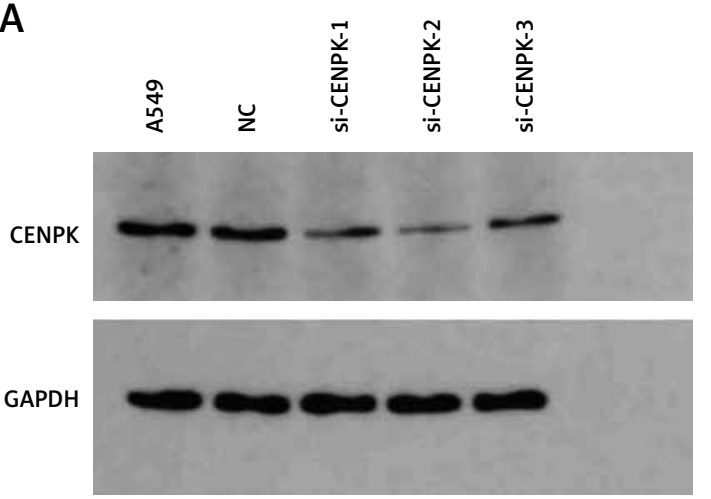

and colony formation assays. The CCK-8 assay showed that after CENPK was silenced by siRNA (si-CENPK group), the proliferation ability of A549 cells was significantly lower than that of the NC group (Figure $8 \mathrm{~A}$ ). The colony formation assay results were consistent with those of the CCK- 8 assay, and the proliferation ability of the si-CENPK group was significantly lower than that of the NC group (Figures 8 B, C).

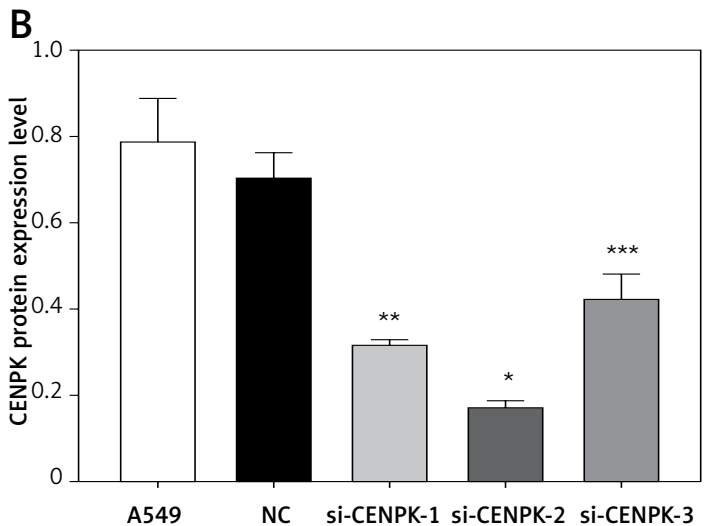

Figure 7. A, B - CENPK protein expression was significantly knocked down by siRNA in the A549 cell line detected by western blotting. ${ }^{*} P<0.05 ;{ }^{* *} p<0.01$

A

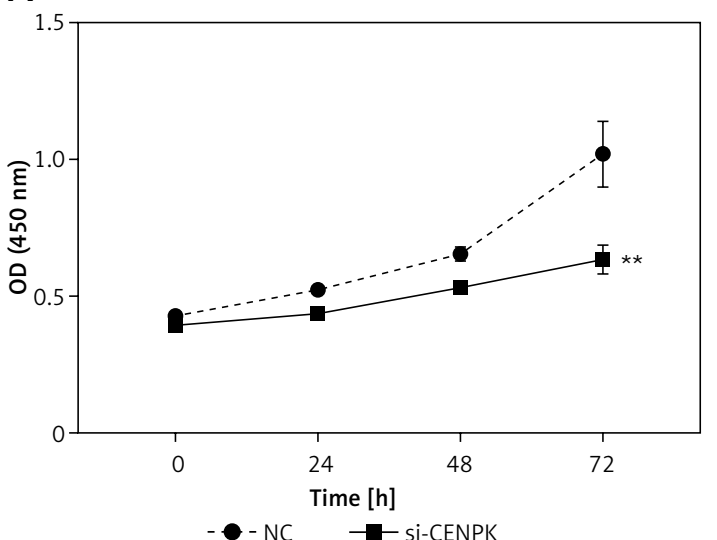

C

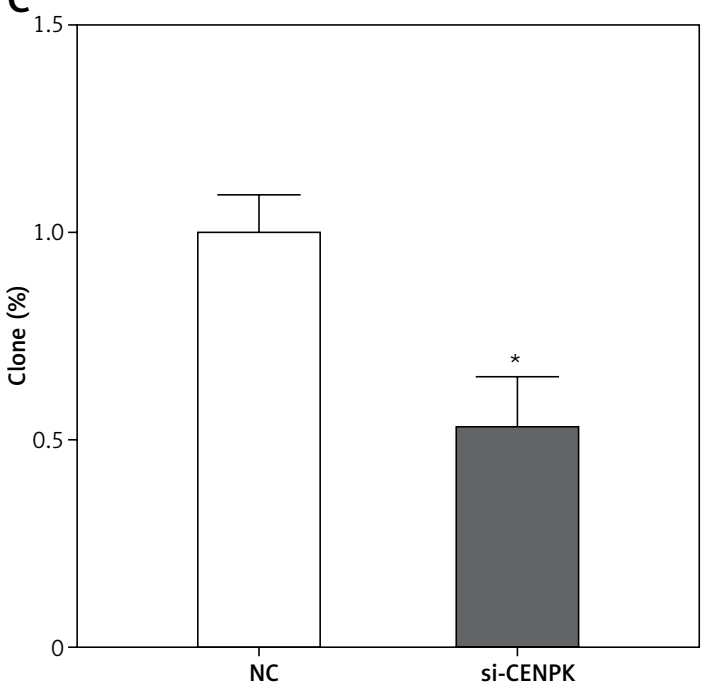

B

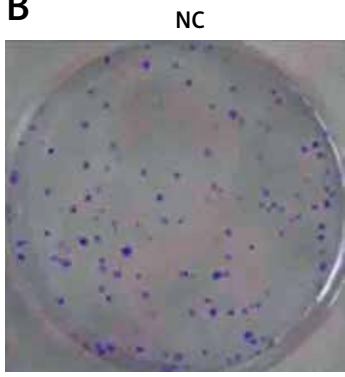

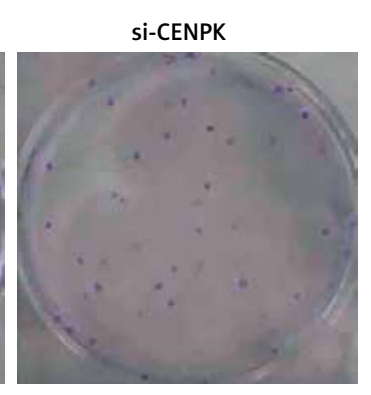

Figure 8. CENPK silencing inhibits the proliferation ability of the A549 cell line. A - CCK-8 assay revealed that CENPK knockdown inhibited proliferation of the A549 cell line. B - Colony formation assays also revealed that CENPK knockdown inhibited proliferation of the $\mathrm{A} 549$ cell line. $\mathrm{C}$ - Statistical analysis of colony formation assays is presented. ${ }^{*} P<0.05 ;{ }^{* *} p<0.01$ 


\section{CENPK silencing inhibits migration and invasion of LUAD cells}

The effect of CENPK silencing on the migration and invasion ability of LUAD cells was examined by wound healing and Transwell assays, following $24 \mathrm{~h}$ of cell culture. The outcomes of the wound healing assay showed that CENPK silencing significantly restrained the migration of LUAD cells (Figures 9 A, B). The results of the Transwell assay showed that both the migration and invasion abilities of the si-CENPK group were lower than those of the NC group (Figures $9 \mathrm{C}, \mathrm{D}$ ).

\section{CENPK silencing attenuates} epithelial-mesenchymal transition (EMT) in LUAD cells

EMT is crucial in the progression of tumor invasion and metastasis [31]. Therefore, the EMT markers (E-cadherin, N-cadherin, Snail and vimentin) were measured by western blotting, which showed that the protein expression level of the epithelial marker (E-cadherin) was significantly higher in the si-CENPK group, while the levels of mesenchymal markers ( $\mathrm{N}$-cadherin, vimentin) and the transcription factor (Snail) were significantly lower than those in the NC group (Figure 10).

\section{CENPK silencing inhibits tumorigenicity of LUAD cells in vivo}

We examined the effect of CENPK silencing on lung tumor growth in vivo. A549 cells were stably infected with lentiviral shRNA-CENPK and implanted into the flank of node mice. The tumor volume was measured every 3 days, with the last measurement on day 30. We found that CENPK silencing in A549 cells significantly reduced tumor volume and tumor weight (Figure 11). The result demonstrated that CENPK reducing could inhibit LUAD growth.

\section{Discussion}

Current research indicates that CENPK as an oncogene plays key roles in many cancers, such as liver cancer and ovarian cancer [16-18]. Howev-
A
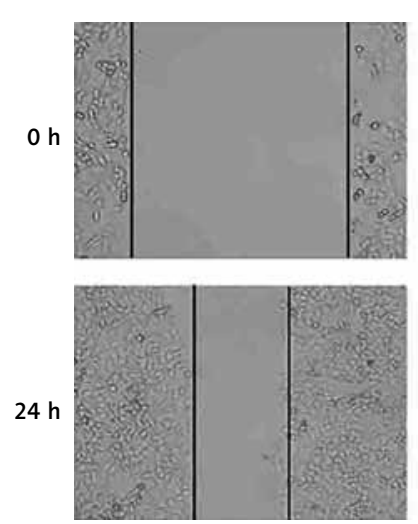

C
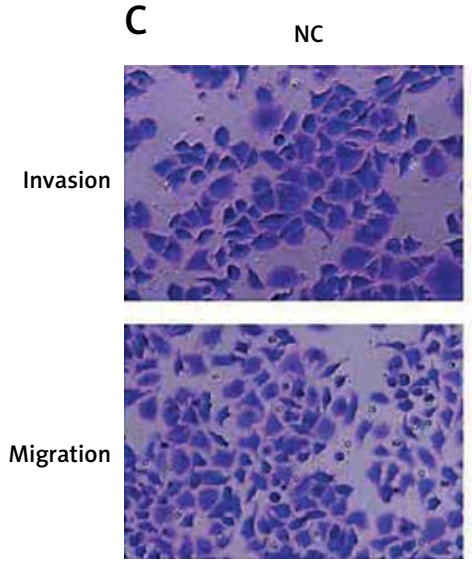

si-CENPK
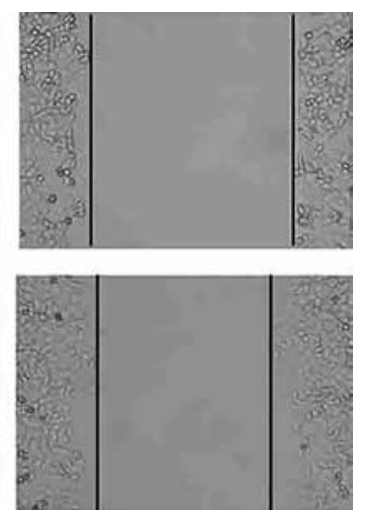

si-CENPK
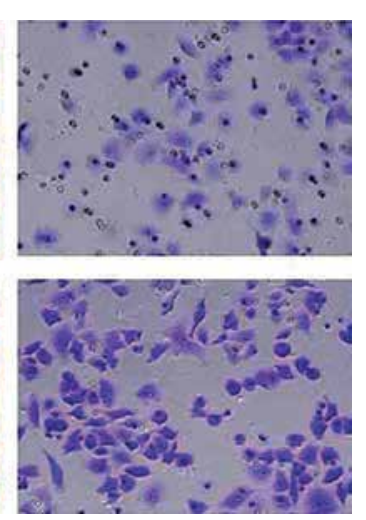

B

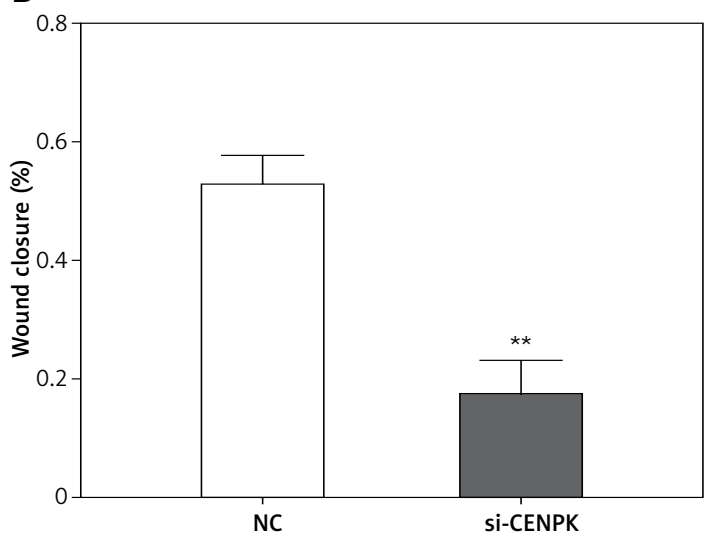

D

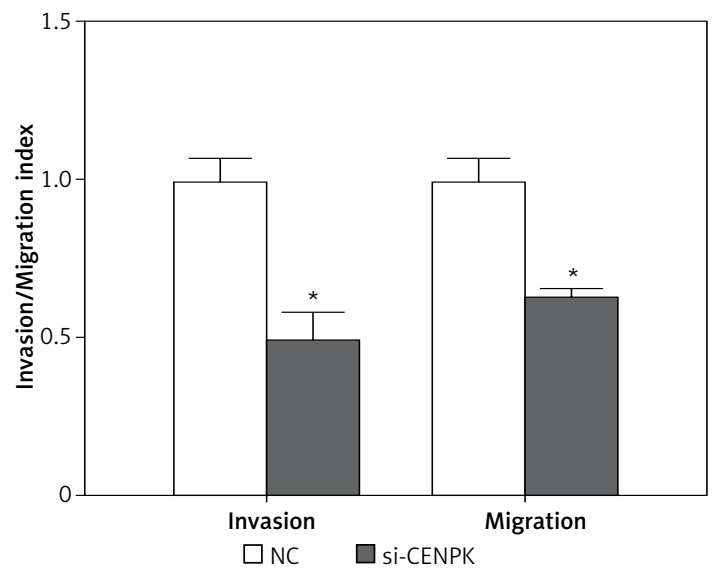

Figure 9. CENPK silencing inhibits migration and invasion of A549 cells. A - Wound healing assay showing that CENPK knockdown decreased the migration ability of the A549 cell line. B - Statistical analysis is presented. $\mathbf{C}$ - Transwell assays showed that CENPK knockdown inhibited migration and invasion of A549 cells. D - Statistical analysis is presented. ${ }^{*} P<0.05 ;{ }^{* *} p<0.01$. 
A

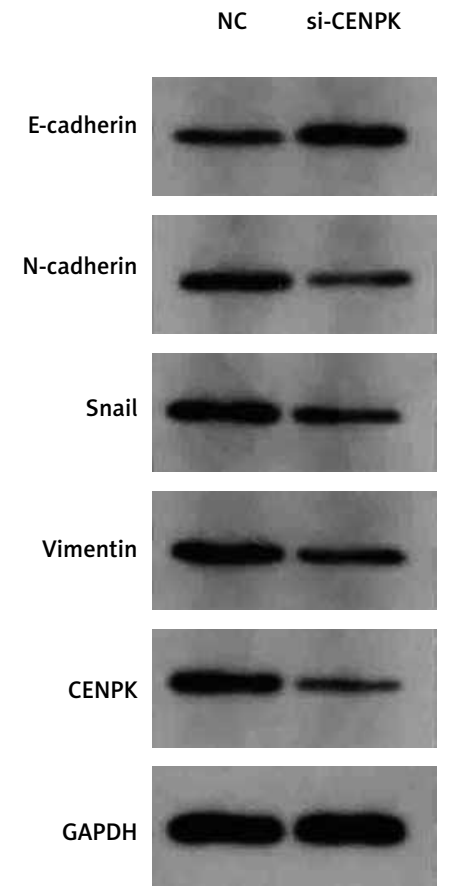

A

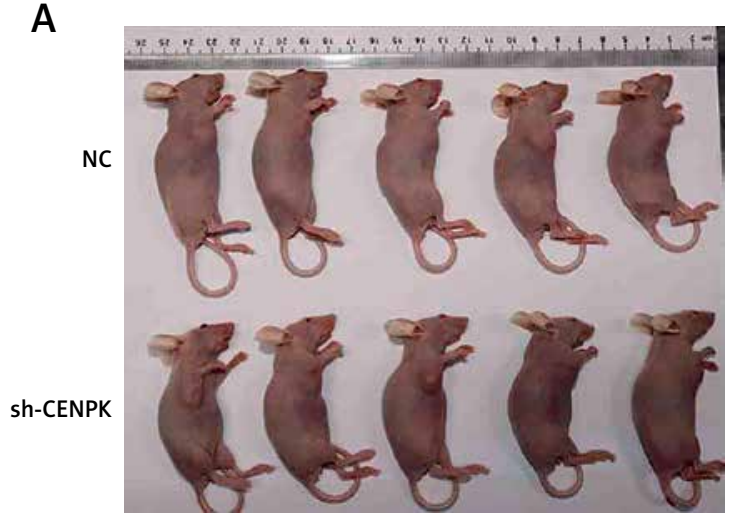

C

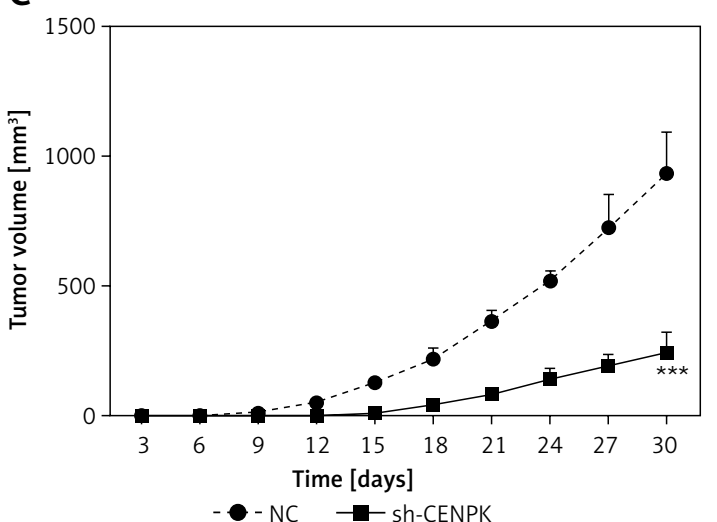

B

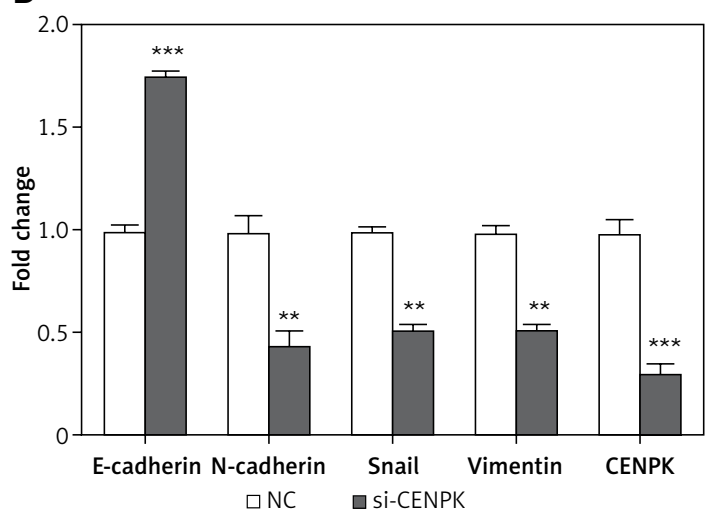

Figure 10. CENPK silencing attenuates EMT-related markers in LUAD cells. A, B - The effect of CENPK silencing on the protein levels of E-cadherin, $\mathrm{N}$-cadherin, Snail, vimentin and CENPK in the A549 cell line detected by western blotting. ${ }^{* *} P<0.01$

\section{B}

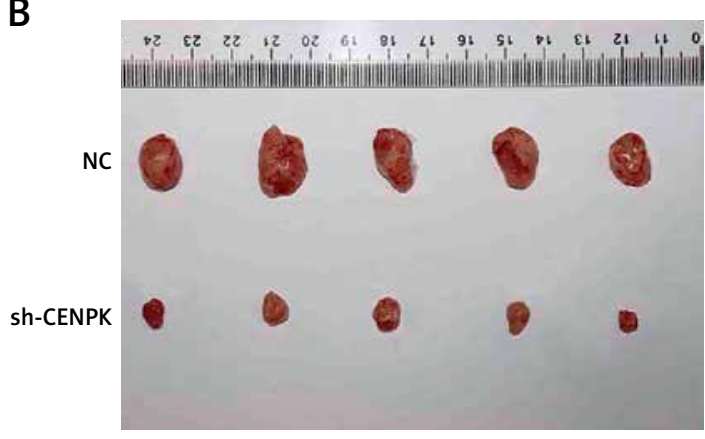

D

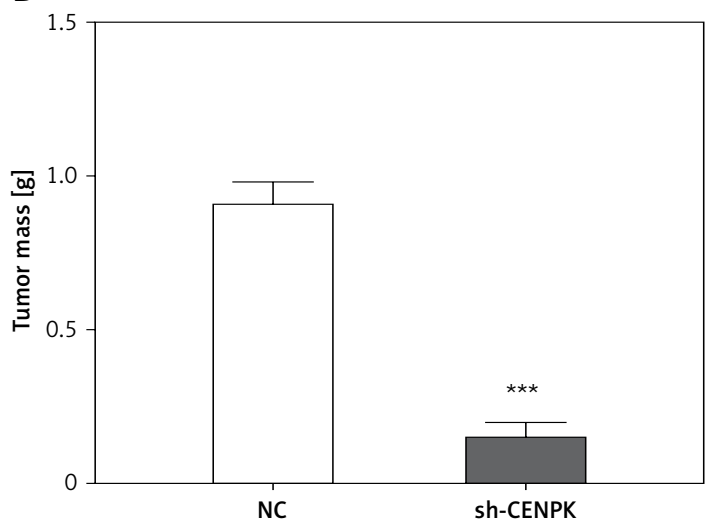

Figure 11. CENPK silencing inhibits tumorigenicity in vivo. A - Subcutaneous tumor transplanted in nude mice after 30 days of inoculation. $\mathbf{B}$ - Tumor exfoliated after the nude mouse was sacrificed. $\mathbf{C}$ - Growth curve of transplanted tumor. D - Comparison of average weight of transplanted tumor in two groups of nude mice $\left({ }^{*} p<0.05 ;{ }^{* *} p<0.01\right.$; ${ }^{* * *} p<0.001 ; \mathrm{NC}-$ negative control) 
er, few reports describe the relationship between CENPK expression and LUAD, which is the main focus of this study.

At present, limited research has shown that CENPK is upregulated in LUAD samples compared to adjacent normal samples and correlated with the poor prognosis of LUAD patients, which is consistent with our research results [32]. In our study, we also found that a high transcript level of CENPK is significantly associated with several clinicopathological features, such as advanced disease stage and $\mathrm{N}$ classifications. Moreover, CENPK is an independent predictor of poor prognosis according to the multivariate Cox regression analysis results. In addition, we constructed an siRNA-mediated CENPK knockdown in vitro and vivo model to further explore the role of CENPK in LUAD, and we found that the proliferation, migration and invasion abilities of the A549 cell line were significantly inhibited by CENPK knockdown.

To date, more than 80 kinds of centromere protein family members have been found, including centromere proteins A, B, C, H, K, I and M [33]. Studies have shown that in addition to CENPK, many other members of the centromeric protein family play a key role in the occurrence and development of various human cancers. CENPF can promote breast cancer bone metastasis by activating the PI3K-AKT-mTORC1 signaling pathway [34]. Downregulation of $\mathrm{CENPH}$ can inhibit the proliferation of renal cell carcinoma and promote cell apoptosis [35]. CENPI is overexpressed in colorectal cancer and acts as an oncogene to promote colorectal cancer cell migration and invasion [36]. CENPA can be used as an independent prognostic factor for LUAD patients, and knocking down CENPA can inhibit proliferation and invasion of LUAD cells $[37,38]$. Research shows that CENPK is a subunit of a CENPH-CENPI-associated centromeric complex that targets CENPA to centromeres and is required for proper kinetochore function and mitotic progression [14]; therefore, CENPK has a strong correlation with CENPA. Based on the above research and the results of this study, we believe that CENPK plays a key role in the development of LUAD.

EMT plays a very important role in embryonic development, organogenesis, wound healing and other physiological processes [39]. In the process of tumor development, activation of EMT increases the migration and invasion characteristics of cancer cells as they shift from the epithelial phenotype to the mesenchymal phenotype, thereby promoting metastasis [40-42]. During the EMT process, E-cadherin, which is one of the most critical cell surface epithelial markers, is downregulated, while mesenchymal markers (vimentin, N-cadherin), and the transcription factor Snail, which regulates EMT, are upregulated; these changes cause loss of cell polarity in epithelial cells, disruption of cell interconnections, and reorganization of the intracellular actin skeleton and also decrease the cell adhesion ability and increase the migration ability $[43,44]$. In addition, studies have shown that vimentin is considered an independent prognostic factor for LUAD and that Snail is related to the clinical outcome of lung cancer [45, 46]. Therefore, these EMT markers were detected for further mechanistic exploration in this study. The results proved that CENPK silencing inhibits proliferation, migration and invasion of the A549 cell line via EMT.

However, there are some limitations to this study. First, only one LUAD cell line (A549) was used for the experiment in this study, and other LUAD cell lines (such as PC-9) were not tested. Second, this study did not further study the role of CENPK by overexpressing CENPK. Last, the results of this study have not been verified by in vivo experiments. Therefore, more in-depth experiments are needed in the future.

In conclusion, CENPK is overexpressed in LUAD and could serve as an independent prognostic factor in LUAD patients. Knockout of CENPK inhibits lung cancer cell proliferation, migration, and invasion by regulating EMT. These findings may provide a new perspective for the clinical treatment of LUAD patients.

\section{Acknowledgments}

We sincerely thank the TCGA database contributors for the high-quality transcript and clinical data.

Fengwen Xie and Bin yuan contributed equally to this study.

\section{Conflict of interest}

The authors declare no conflict of interest.

\section{References}

1. Siegel RL, Miller KD, Jemal A. Cancer statistics, 2020. CA Cancer J Clin 2020; 70: 7-30.

2. Chen W, Zheng R, Baade PD, et al. Cancer statistics in China, 2015. CA Cancer J Clin 2016; 66: 115-32.

3. Chen W, Zheng R, Zhang S, et al. Cancer incidence and mortality in China, 2013. Cancer Lett 2017; 401: 63-71.

4. Seow WJ, Matsuo K, Hsiung CA, et al. Association between GWAS-identified lung adenocarcinoma susceptibility loci and EGFR mutations in never-smoking Asian women, and comparison with findings from Western populations. Hum Mol Genet 2017; 26: 454-65.

5. Ma J, Chen X, Lin M, et al. Bioinformatics analysis combined with experiments predicts CENPK as a potential prognostic factor for lung adenocarcinoma. Cancer Cell Int 2021; 21: 65.

6. Hawkins P, Stevenson T, Powari M. Use of the idylla EGFR mutation test for variant detection in non-small 
cell lung cancer samples. Am J Clin Pathol 2021; doi: 10.1093/ajcp/aqab003.

7. Meng Y, Qian X, Zhao L, et al. Trichostatin A downregulates bromodomain and extra-terminal proteins to suppress osimertinib resistant non-small cell lung carcinoma. Cancer Cell Int 2021; 21: 216.

8. Nghiem P, Bhatia S, Lipson EJ, et al. Three-year survival, correlates and salvage therapies in patients receiving first-line pembrolizumab for advanced Merkel cell carcinoma. J Immunother Cancer 2021; 9: e002478.

9. Wang DD, Shaver LG, Shi FY, et al. Comparative efficacy and safety of PD-1/PD-L1 immunotherapies for nonsmall cell lung cancer: a network meta-analysis. Eur Rev Med Pharmacol Sci 2021; 25: 2866-84.

10. Li B, Jiang C, Pang L, et al. Toxicity profile of combining PD-1/PD-L1 inhibitors and thoracic radiotherapy in non-small cell lung cancer: a systematic review. Front Immunol 2021; 12: 627197.

11. Dall'Olio FG, Gelsomino F, Conci N, et al. PD-L1 expression in circulating tumor cells as a promising prognostic biomarker in advanced non-small-cell lung cancer treated with immune checkpoint inhibitors. Clin Lung Cancer 2021. doi: 10.1016/j.cllc.2021.03.005.

12. Mao Y, Yang D, He J, Krasna MJ. Epidemiology of lung cancer. Surg Oncol Clin N Am 2016; 25: 439-45.

13. Foltz DR, Jansen LE, Black BE, et al. The human CENP-A centromeric nucleosome-associated complex. Nat Cell Biol 2006; 8: 458-69.

14. Okada M, Cheeseman IM, Hori T, et al. The CENP-H-I complex is required for the efficient incorporation of newly synthesized CENP-A into centromeres. Nat Cell Biol 2006; 8: 446-57.

15. Izuta $H$, Ikeno $M$, Suzuki N, et al. Comprehensive analysis of the ICEN (Interphase Centromere Complex) components enriched in the CENP-A chromatin of human cells. Genes Cells 2006; 11: 673-84.

16. Wang J, Li H, Xia C, et al. Downregulation of CENPK suppresses hepatocellular carcinoma malignant progression through regulating YAP1. Onco Targets Ther 2019 12: 869-82.

17. Wang H, Liu W, Liu L, et al. Overexpression of centromere protein $\mathrm{K}(\mathrm{CENP}-\mathrm{K})$ gene in hepatocellular carcinoma promote cell proliferation by activating AKT/TP53 signal pathway. Oncotarget 2017; 8: 73994-4005.

18. Lee YC, Huang CC, Lin DY, Chang WC, Lee KH. Overexpression of centromere protein $\mathrm{K}$ (CENPK) in ovarian cancer is correlated with poor patient survival and associated with predictive and prognostic relevance. Peer J 2015; 3: e1386.

19. Komatsu M, Yoshimaru T, Matsuo T, et al. Molecular features of triple negative breast cancer cells by genome-wide gene expression profiling analysis. Int J Oncol 2013; 42: 478-506.

20. Jia D, Park JH, Kaur H, et al. Towards decoding the coupled decision-making of metabolism and epithelial-to-mesenchymal transition in cancer. $\mathrm{Br} J$ Cancer 2021; 124: 1902-11.

21. Chaudhary S, Pothuraju R, Rachagani S, et al. Dua blockade of EGFR and CDK4/6 delays head and neck squamous cell carcinoma progression by inducing metabolic rewiring. Cancer Lett 2021; 510: 79-92.

22. Zhou Y, Hill C, Yao L, et al. Quantitative proteomic analysis in alveolar type ii cells reveals the different capacities of RAS and TGF- $\beta$ to induce epithelial-mesenchymal transition. Front Mol Biosci 2021; 8: 595712.

23. Li CL, Li J, Gong SY, et al. Targeting the ILK/YAP axis by LFG-500 blocks epithelial-mesenchymal transition and metastasis. Acta Pharmacol Sin 2021. doi: 10.1038/ s41401-021-00655-y.

24. He C, Ali DJ, Aseervatham GS, Sun B, Xiao Z. Microvesicles - promising tiny players' of cancer stem cells targeted liver cancer treatments: the interesting interactions and therapeutic aspects. Pharmacol Res 2021; 169: 105609.

25. Zhang L, Liu Z, Zhu J. In silico screening using bulk and single-cell RNA-seq data identifies RIMS2 as a prognostic marker in basal-like breast cancer: a retrospective study. Medicine 2021; 100: e25414.

26. Saitoh M. Involvement of partial EMT in cancer progression. J Biochem 2018; 164: 257-64.

27. Tomczak K, Czerwińska P, Wiznerowicz M. The Cancer Genome Atlas (TCGA): an immeasurable source of knowledge. Contem Oncol 2015; 19: A68-77.

28. Rhodes DR, Yu J, Shanker K, et al. ONCOMINE: a cancer microarray database and integrated data-mining platform. Neoplasia 2004; 6: 1-6.

29. Mizuno H, Kitada K, Nakai K, Sarai A. PrognoScan: a new database for meta-analysis of the prognostic value of genes. BMC Med Genomics 2009; 2: 18.

30. Zeng SG, Lin X, Liu JC, Zhou J. Hypoxia-induced internalization of connexin 26 and connexin 43 in pulmonary epithelial cells is involved in the occurrence of non-small cell lung cancer via the P53/MDM2 signaling pathway. Int J Oncol 2019; 55: 845-59.

31. Lamouille $S, X u$ J, Derynck R. Molecular mechanisms of epithelial-mesenchymal transition. Nat Rev Mol Cell Biol 2014; 15: 178-96.

32. Yimin L, Xihua W, Li L, et al. Bioinformatics analysis reveals centromere protein $\mathrm{K}$ can serve as potential prognostic biomarker and therapeutic target for non-small cell lung cancer. Curr Bioinform 2020; 15: 1-14.

33. McKinley KL, Cheeseman IM. The molecular basis for centromere identity and function. Nat Rev Mol Cell Biol 2016; 17: 16-29.

34. Sun J, Huang J, Lan J, et al. Overexpression of CENPF correlates with poor prognosis and tumor bone metastasis in breast cancer. Cancer Cell Int 2019; 19: 264.

35. Wu X, Lin Y, Shi L, et al. Upregulation of centromere protein $\mathrm{H}$ is associated with progression of renal cell carcinoma. J Mol Histol 2015; 46: 377-85.

36. Ding N, Li R, Shi W, He C. CENPI is overexpressed in colorectal cancer and regulates cell migration and invasion. Gene 2018; 674: 80-6.

37. Wu Q, Qian YM, Zhao XL, et al. Expression and prognostic significance of centromere protein $A$ in human lung adenocarcinoma. Lung Cancer 2012; 77: 407-14.

38. Wu Q, Chen YF, Fu J, et al. Short hairpin RNA-mediated down-regulation of CENP-A attenuates the aggressive phenotype of lung adenocarcinoma cells. Cell Oncol 2014; 37: 399-407.

39. Thiery JP, Acloque H, Huang RY, Nieto MA. Epithelialmesenchymal transitions in development and disease. Cell 2009; 139: 871-90.

40. Yuanhua L, Pudong Q, Wei Z, et al. TFAP2A Induced KRT16 as an oncogene in lung adenocarcinoma via EMT. Int J Biol Sci 2019; 15: 1419-28.

41. Boulding T, McCuaig RD, Tan A, et al. LSD1 activation promotes inducible EMT programs and modulates the tumour microenvironment in breast cancer. Sci Rep 2018; 8: 73.

42. Zhou P, Li B, Liu F, et al. The epithelial to mesenchymal transition (EMT) and cancer stem cells: implication for treatment resistance in pancreatic cancer. Mol Cancer 2017; 16: 52. 
43. Moustakas A, Heldin CH. Signaling networks guiding epithelial-mesenchymal transitions during embryogenesis and cancer progression. Cancer Sci 2007; 98: 1512-20.

44. Raoof S, Mulford IJ, Frisco-Cabanos H, et al. Targeting FGFR overcomes EMT-mediated resistance in EGFR mutant non-small cell lung cancer. Oncogene 2019; 38: 6399-413.

45. Groeneveld S, Faget J, Zangger N, Meylan E. Snail mediates repression of the Dlk1-Dio3 locus in lung tumor-infiltrating immune cells. Oncotarget 2018; 9: 32331-45.

46. Bian T, Zheng L, Jiang $D$, et al. Overexpression of fibronectin type III domain containing $3 \mathrm{~B}$ is correlated with epithelial-mesenchymal transition and predicts poor prognosis in lung adenocarcinoma. Exp Ther Med 2019; 17: 3317-26. 